\section{Method for real-time monitoring of protein degradation at the single cell level}

\author{
Lijuan Zhang ${ }^{1}$, Nadya G. Gurskaya', Ekaterina M. Merzlyak², Dmitry B. \\ Staroverov², Nikolay N. Mudrik¹, Olga N. Samarkina ${ }^{3}$, Leonid M. Vinokurov³, \\ Sergey Lukyanov'1, and Konstantin A. Lukyanov ${ }^{1}$ \\ ${ }^{1}$ Institute of Bioorganic Chemistry, Moscow, ${ }^{2}$ Evrogen JSC, Moscow, and Institute of \\ Bioorganic Chemistry, Pushchino Branch, Pushchino, Russia
}

BioTechniques 42:446-450 (April 2007)

doi 10.2144/000112453

Protein degradation is important in practically all aspects of cellular physiology (1). Together with transcription and translation, proteolysis ensures maintenance and rapid regulation of individual protein concentration in living cells. The half-life for different proteins varies between a few minutes and days. Moreover, decay rate for many proteins change drastically throughout the cell cycle or in response to external stimuli.

Two methods are commonly used to determine a protein's half-life, namely radioactive pulse-chase analysis and cycloheximide chase (2). Pulse-chase analysis provides minimal distortion of normal cell physiology. The main disadvantages of this method are its laboriousness and necessity for radiolabeling. In contrast to pulse-chase analysis, cycloheximide chase strongly affects cellular metabolism that should be considered a serious disadvantage for this approach. Importantly, both methods do not allow real-time measurements at the single cell level.

The use of green fluorescent protein (GFP) provided an opportunity to apply fluorescence microscopy and flow cytometry to protein degradation analysis (3-6). However, to extract information regarding protein degradation using GFP, one should block the synthesis of new GFP molecules (e.g., by cycloheximide) $(3,4)$. Even in presence of cycloheximide, residual GFP chromophore maturation affects estimation of degradation rate, especially when maturation and degradation half-times are comparable.

Since 2002, a number of so-called photoactivatable fluorescent proteins (PAFPs) have been developed (7). Most commonly, local PAFP activation is used to visualize protein movement within cells. Here we propose to use PAFP activation within a whole cell to monitor protein degradation (Figure 1). Indeed, while steady-state fluorescence intensity of an FP-tagged protein depends on both synthesis and degradation rates, photoactivation creates a fluorescent signal that depends only on protein degradation (fluorescence bleaching during observation should be taken into account and made allowance for). Thus, timelapse imaging of activated PAFP allows quantification of the tagged protein degradation process. Also, this protocol can be potentially adapted for PAFP photoactivation in large cell culture samples and their further analysis by flow cytometry or microplate readers.

To demonstrate the usability of the method proposed, we used the green-tored photoconvertible fluorescent protein Dendra2 (Evrogen, Moscow, Russia), which is a commercially available improved version of Dendra (8). In the dark, Dendra2 matures up to the green fluorescent state with excitationemission maxima at 490 and $507 \mathrm{~nm}$, respectively. Upon maturation, it can be converted into a red-emitting protein (excitation-emission at $553 / 573 \mathrm{~nm}$ ) by irradiation with violet (e.g., $405 \mathrm{~nm}$ ) or blue (e.g., $488 \mathrm{~nm}$ ) light. Due to its monomeric state, Dendra2 can be safely used for protein labeling. In contrast to other PAFPs, Dendra2 can be activated with blue light, which is less damaging compared with ultraviolet (UV) or violet light. Using a well-established assay based on dithionite reduction of chromophore of urea-denatured fluorescent protein followed by dilution of the sample and maturation of the fluorescent protein starting from the native polypeptide (9), we measured Dendra2 maturation halftime as $90 \mathrm{~min}$ at $37^{\circ} \mathrm{C}$.

Similar to GFP, Dendra2 was found to be a long-lived protein. In $\mathrm{HeLa}$ and HEK 293 cells, we observed no decrease in Dendra2 green fluorescence for several hours after the addition of cycloheximide (not shown). To photoconvert Dendra2 throughout the experiments, we applied 10-20 s of irradiation with blue light from a 100 W Hg-lamp using the GFP filter set. As a result, the green signal decreased while clearly detectable red fluorescence appeared. Then, we monitored red fluorescence at $37^{\circ} \mathrm{C}$ in the confocal mode (Leica DMIRE2 TCS SP2 microscope; Leica Microsystems $\mathrm{GmbH}$, Wetzlar, Germany) using the 543-nm laser line. Practically no decay of red fluorescence after Dendra2 photoconversion was observed (Figure 2A), demonstrating very high stability of this protein in living cells. Next we tested the influence of peptides or proteins known to determine fast degra-

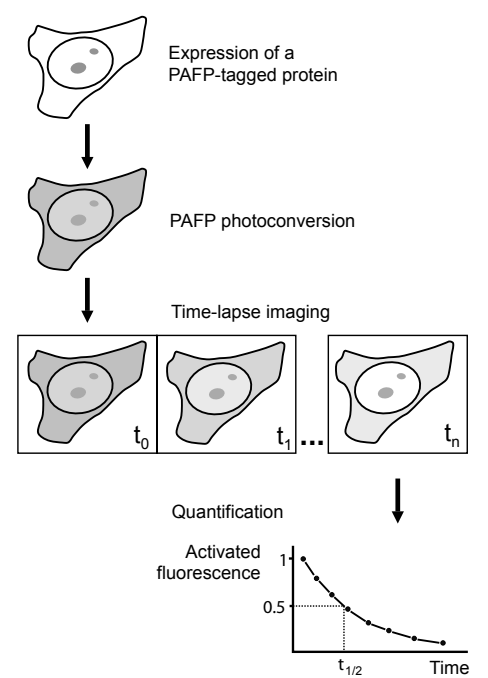

Figure 1. Outline of the proposed method Cells are transfected with a construct coding for target protein fused with a photoactivatable fluorescent proteins (PAFP). A steady-state concentration of the fusion protein and corresponding fluorescent signal (e.g., green) depends on protein synthesis and maturation rates as well as protein degradation rate. After a photoconversion of the PAFP in a whole cell, a pool of distinct fluorescent molecules (e.g., red) appears, which is independent from synthesis and maturation of the new PAFP molecules. Thus, the decay of the activated fluorescence directly corresponds to the degradation of the PAFP-tagged protein. Timelapse imaging of the activated signal allows for quantification of degradation process in real-time at the single cell level. 


\section{Benchmarks}

dation on Dendra2 stability. First, we attached the PEST motif of ornithine decarboxilase (ODC), which should cause rapid degradation of the protein via an unusual ubiquitine-independent antizyme-controlled pathway (10). It was reported that GFP fused to PEST $\mathrm{ODC}_{\mathrm{ODC}}$ possesses a greatly reduced half-life, although the exact value varies from 2 (3) to $9.8 \mathrm{~h}$ (5), depending on the exact fusion structure and cell line type. In the present work, we constructed Dendra2 fused with residues 422-461 of mouse ODC similarly to destabilized enhanced GFP (dEGFP) (3). Dendra2-PEST ${ }_{\text {ODC }}$ showed fast decay of the activated red signal with a half-life of 2-3 h (Figure 2A). Also, Dendra2 fused with PEST motif of neural proliferation and differentiation control protein-1 (NPDC1) (11) in PC12 cells demonstrated very dim green fluorescence that disappeared with a half-life of 15 min after the addition of $100 \mu \mathrm{g} / \mathrm{mL}$ cycloheximide (not shown). Then we evaluated Dendra2 fused to an uncleavable mutant of ubiquitin (Ub-G75A/G76VDendra2), which should be degraded very rapidly. Indeed, cells expressing this construct demonstrated no green fluorescence, but they readily became fluorescent after incubation with 0.5 $\mu \mathrm{M}$ proteosome inhibitor epoxomicin (not shown). These results clearly demonstrated that Dendra2, similar to GFP (3-5), is a very stable protein itself, does not interfere with degradation of fusion peptides and proteins, and can be effectively targeted to the proteasomal degradation pathway.

To further test the applicability of the proposed method, we fused Dendra2 with $I \kappa B \alpha$, a well-characterized protein having relatively fast and regulatable decay in cells $(4,12-14)$. Cells with moderate expression levels of IкB $\alpha$ Dendra2 demonstrated the expected, predominantly cytoplasmic, localization of green fluorescence (Figure 2B). After photoconversion, time-lapse series showed fast decay of the red signal with a half-life of 1.5-2 $\mathrm{h}$. The addition of a proteasome inhibitor immediately terminated red fluorescence decay (Figure 2C). Thus, the decrease of red fluorescent signal was caused by proteasomal degradation of the fusion protein. The rate of red signal decay was in good agreement with the available data

A
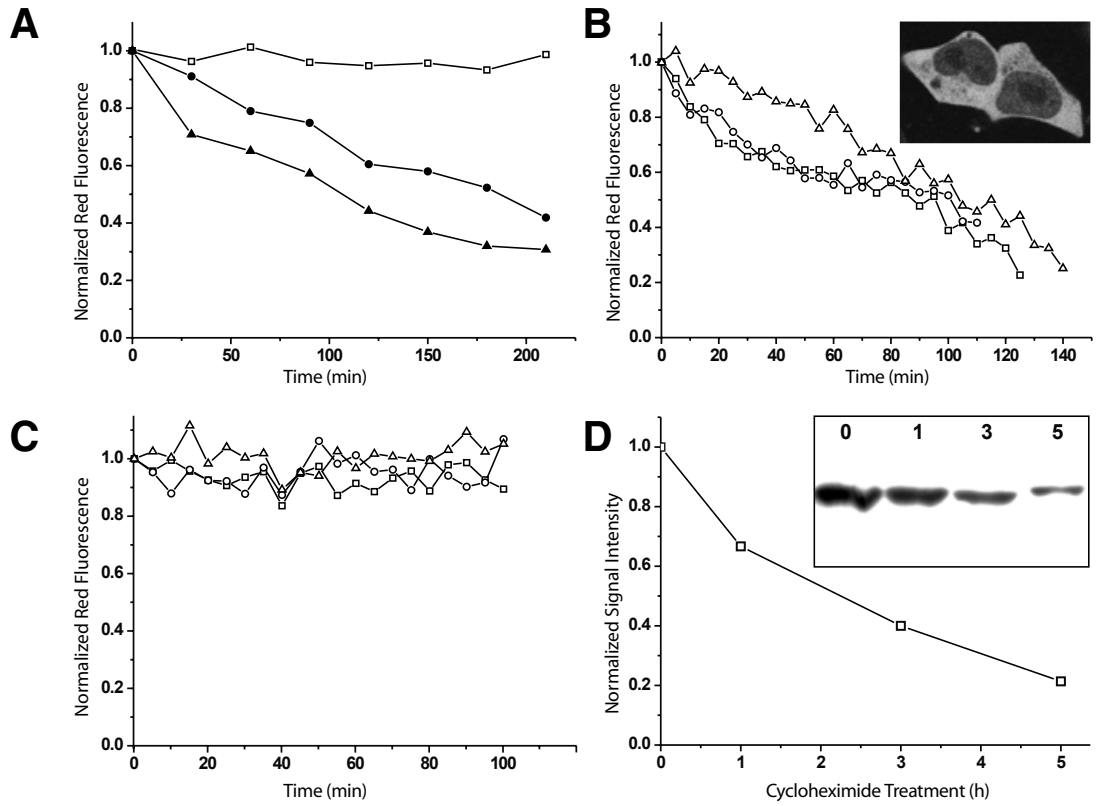

E
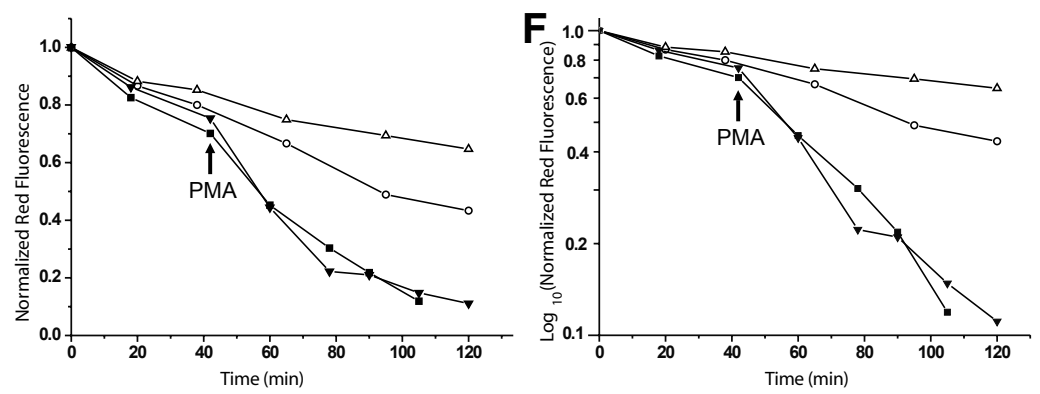

Figure 2. Monitoring protein degradation using Dendra2 in transiently transfected HEK 293 Phoenix Eco cells. (A) Time course of red fluorescence change in cells transfected with Dendra2 (open squares) or destabilized Dendra2-PEST ${ }_{\mathrm{ODC}}$ (filled circles and triangles). Here and in panels B, C, E, and $\mathrm{F}$, each line corresponds to a representative individual cell (10-15 cells were measured for each experiment). (B) Time course of red fluorescence change in cells expressing I $\kappa \mathrm{B} \alpha$-Dendra2. Inset shows an example of a confocal image of the cells, excitation at $488 \mathrm{~nm}$, detection at 500-530 $\mathrm{nm}$. (C) The same dish as in panel B, but immediately after addition of $0.5 \mu \mathrm{M}$ proteosome inhibitor MG132. (D) I $\mathrm{BB} \alpha-$ Dendra2 decay as measured by cycloheximide chase. Inset shows immunoblotting using antibodies against Dendra2 of I $\mathrm{BB} \alpha$-Dendra2-transtected cells treated with cycloheximide for $0,1,3$, and $5 \mathrm{~h}$. The band shown is about $60 \mathrm{kDa}$, as expected for the fusion protein, and no other bands were detected. The graph shows quantification of the immunoblotting. (E) Decay of red fluorescence from I $\kappa \mathrm{B} \alpha$-Dendra2 (resting cells, open symbols; cells treated with $0.1 \mu \mathrm{g} / \mathrm{mL}$ PMA at a time point designated by arrow, filled symbols). (F) The same data as in panel E, but on a semi-logarithmic scale. Before the time-lapse imaging in each experiment, we controlled fluorescence bleaching using immediate repeated laser scanning. Conditions with negligible bleaching after 20 scans were used. ODC, ornithine decarboxilase; PMA, phorbol 12-myristate 13-acetate.

on the half-life of IкB $\alpha$-EGFP (4) and I $\mathrm{B} \alpha$ alone (12). The directly measured I $\kappa \mathrm{B} \alpha$-Dendra2 half-life using cycloheximide chase and polyclonal antibodies against Dendra2 also equaled $2 \mathrm{~h}$ (Figure 2D). As an additional control, we studied Dendra2 fused to long-lived mutant I $\mathrm{KB} \alpha$ carrying substitutions S32A and S36A, which eliminate sites for its phosphorylation $(4,13,14)$. As expected, no red fluorescence decay was observed for I $\mathrm{KB} \alpha(\mathrm{S} 32 \mathrm{~A} / \mathrm{S} 36 \mathrm{~A})$ -
Dendra2 (not shown). It was shown earlier that the phorbol ester, phorbol 12-myristate 13-acetate (PMA), increases the $\mathrm{I} \kappa \mathrm{B} \alpha$ degradation rate $(4,14)$. Indeed, a considerable acceleration of red fluorescence decay after cell treatment with PMA was detected using photoactivation of IKB $\alpha$-Dendra2 (Figure 2, E and F). I $\kappa \mathrm{B} \alpha$ half-life of 20 min was estimated for PMA-treated cells; it is fully consistent with the corresponding 15-min decay rate described 


\section{Benchmarks}

earlier $(4,14)$. Importantly, we were able to detect a change in the degradation rate in individual cells, as the same cells were monitored both before and after treatment. To summarize, the degradation behavior of I $\mathrm{KB} \alpha$-Dendra2 monitored by red fluorescence decay after Dendra2 photoactivation was very similar to that of endogenous $\mathrm{I} \kappa \mathrm{B} \alpha$ as well as exogenous I $\mathrm{K} B \alpha$-EGFP fusion.

Apparent disadvantages of the proposed methods should be noted. First, one has to work with chimeric proteins, which are often overexpressed to clearly detect a fluorescent signal. Second, PAFP fusion can potentially affect target protein degradation, and this should be carefully controlled. Also, target proteins with very fast basic turnover are inaccessible for this method, because a PAFP has no time to mature in this case. However, all these limitations are common for protein labeling with GFP, which is nevertheless widely used in modern cell biology. At the same time, our method provides a unique opportunity to observe real-time target protein degradation at the single cell level without addition of protein synthesis inhibitors, to follow conditionally dependent changes in degradation rate, and to correlate this with cell cycle stage, morphology, and other characteristics of the particular cells.

\section{ACKNOWLEDGMENTS}

This work was supported by grants from the European Commission FP-6 Integrated Project LSHG-CT-2003503259, the Russian Academy of Sciences for the program Molecular and Cell Biology, grant GM070358 from the National Institutes of Health, and Russian Science Support Foundation to K.A.L.

\section{COMPETING INTERESTS STATEMENT}

Dendra2 is sold by Evrogen JSC, Moscow, Russia. N.G.G., E.M.M., D.B.S., N.N.M., S.L., and K.A.L., have interest in Evrogen JSC. E.M.M. and D.B.S. are employed by Evrogen JSC.

\section{REFERENCES}

1. Varshavsky, A. 2005. Regulated protein degradation. Trends Biochem. Sci. 30:283-286.
2.Zhou, P. 2004. Determining protein halflives. Methods Mol. Biol. 284:67-77.

3.Li, X., X. Zhao, Y. Fang, X. Jiang, T. Duong, C. Fan, C.C. Huang, and S.R. Kain. 1998. Generation of destabilized green fluorescent protein as a transcription reporter. J. Biol. Chem. 273:34970-34975.

4. Li, X., Y. Fang, X. Zhao, X. Jiang, T. Duong, and S.R. Kain. 1999. Characterization of NFkappaB activation by detection of green fluorescent protein-tagged IkappaB degradation in living cells. J. Biol. Chem. 274:2124421250

5.Corish, P. and C. Tyler-Smith. 1999. Attenuation of green fluorescent protein half-life in mammalian cells. Protein Eng. 12:1035-1040.

6. Dantuma, N.P., K. Lindsten, R. Glas, M. Jellne, and M.G. Masucci. 2000. Short-lived green fluorescent proteins for quantifying ubiquitin/proteasome-dependent proteolysis in living cells. Nat. Biotechnol. 18:538-543.

7.Lukyanov, K.A., D.M. Chudakov, S. Lukyanov, and V.V. Verkhusha. 2005. Photoactivatable fluorescent proteins. Nat. Rev. Mol. Cell Biol. 6:885-891.

8.Gurskaya, N.G., V.V. Verkhusha, A.S. Shcheglov, D.B. Staroverov, T.V. Chepurnykh, A.F. Fradkov, S. Lukyanov, and K.A. Lukyanov. 2006. Engineering of a monomeric green-to-red photoactivatable fluorescent protein induced by blue light. Nat. Biotechnol. 24:461-465.

9. Reid, B.G. and G.C. Flynn. 1997. Chromophore formation in green fluorescent protein. Biochemistry 36:6786-6791.

10. Pegg, A.E. 2006. Regulation of ornithine decarboxylase. J. Biol. Chem. 281:1452914532.

11.Spencer, M.L., M. Theodosiou, and D.J. Noonan. 2004. NPDC-1, a novel regulator of neuronal proliferation, is degraded by the ubiquitin/proteasome system through a PEST degradation motif. J. Biol. Chem. 279:3706937078 .

12. Henkel, T., T. Machleidt, I. Alkalay, M. Kronke, Y. Ben-Neriah, and P.A. Baeuerle. 1993. Rapid proteolysis of I kappa B-alpha is necessary for activation of transcription factor NF-kappa B. Nature 365:182-185.

13. Brown, K., S. Gerstberger, L. Carlson, G. Franzoso, and U. Siebenlist. 1995. Control of I kappa B-alpha proteolysis by site-specific, signal-induced phosphorylation. Science 267:1485-1488

14. Ghoda, L., X. Lin, and W.C. Greene. 1997. The 90-kDa ribosomal S6 kinase pp90rsk phosphorylates the $\mathrm{N}$-terminal regulatory domain of IkappaBalpha and stimulates its degradation in vitro. J. Biol. Chem. 272:21281-21288.

Received 17 January 2007; accepted 2 March 2007.

Address correspondence to Konstantin A. Lukyanov, Institute of Bioorganic Chemistry, Miklukho-Maklaya 16/10, 117997 Moscow, Russia.e-mail:kluk@ibch.ru

To purchase reprints of this article, contact: Reprints@BioTechniques.com 